Juan A. Fargallo $\mathbb{a}$ Jose A. Davila $\mathbb{J}$ Jaime Potti

Ana de Leon $₫$ Vicente Polo

\title{
Nest size and hatchling sex ratio in chinstrap penguins
}

\begin{abstract}
Variation in the sex ratio at hatching in the chinstrap penguin Pygoscelis antarctica was investigated, using molecular sexing to test predictions of sex allocation theory. The sex ratio was slightly male-biased (0.54) but did not differ significantly from parity. The proportion of males increased with nest size, an estimator of parental quality in chinstrap penguins. Highquality parents were able to produce and rear a higher proportion of male offspring, the more costly sex in this slightly sexually dimorphic species. Our results may be in agreement with Trivers and Willard's (1973) argument on biases in the offspring sex ratio being contingent on parental condition or quality.
\end{abstract}

\section{Introduction}

Sex allocation theory predicts that individuals are expected to vary the sex ratio of their offspring in relation to the specific fitness benefits of sons and daughters (Hamilton 1967; Trivers and Willard 1973; Charnov 1982). Since the development of modern molecular

\footnotetext{
J. A. Fargallo (\&) \& V. Polo

Departamento de Ecologia Evolutiva, Museo Nacional de Ciencias Naturales (CSIC), J. Gutierrez Abascal 2, 28006 Madrid, Spain

E-mail: fargallo@mncn.csic.es

Tel: +34-91-4111328

Fax: +34-91-5645078

J. A. Davila

Instituto de Investigacion en Recursos Cinegeticos (CSIC-UCLM), Ronda de Toledo s/n, 13005 Ciudad Real, Spain

J. Potti

Departamento de Biologia Aplicada, Estacion Biologica de Donana, Apartado 1056,

41080 Sevilla, Spain

A. de Leon

Ornithology Group, Institute of Biomedical and Life Sciences,

Graham Kerr Building, University of Glasgow, Glasgow, G12 8QQ, UK
}

sexing techniques in birds (Griffiths and Tiwari 1995), a sizeable number of studies in birds have related variation in brood sex ratios with the amount of care that parents directly (parental quality) or indirectly (environmental and social factors) invest in their offspring (see Hasselquist and Kempenaers 2002; Komdeur and Pen 2002 for reviews). However, some other studies have failed to find such relationships (e.g. Newton and Marquiss 1979; Koenig and Dickinson 1996; Bradbury et al. 1997; Westerdahl et al. 1997; Plagiani et al. 1999). A common problem in many of these studies is that only sex ratios at fledging could be recorded, preventing researchers from assessing the importance of pre-fledging sex-biased mortality. More studies on offspring sex ratios before mortality occurs will be necessary to understand the apparent adaptive nature of avian sex-ratio manipulation (Sheldon 1998).

One hypothesis on sex allocation theory posits that when the relative fitness of sons and daughters is dependent on parental quality, i.e. amount and suitability of parental resources, and the reproductive value differs between the sexes, parents should produce more individuals of that sex that maximises their own fitness under the conditions in which they are living (Trivers and Willard 1973). This is expected in polygynous species in which males experience a large among-individual variance in reproductive success.

In this study, we investigated the sex ratio at hatching in a colony of chinstrap penguins (Pygoscelis antarctica). Our aim was reporting for the first time the sex ratio at birth in a penguin species and look for its relationships with surrogates of parental quality. As indicators of parental quality, we used: (1) nest size (measured as nest weight), (2) body size (measured as flipper length), and (3) breeding time (expressed as hatching date). Selection of these parental attributes as indicators of parental quality was based on previous studies on breeding performance of chinstrap penguins. (1) Nests of pygoscelid penguins consist of piles of stones accumulated in scrapes on the ground (Muller-Schwarze 1984). The size of their nest is a property that chinstrap-penguin parents con- 
tinuously monitor throughout the incubation and brooding periods (Carrascal et al. 1995; Moreno et al. 1995), reacting to changes in it by appropriate changes in the amount of stone collecting (Moreno et al. 1999; Fargallo et al. 2001).Penguins may spend several hours collecting stones from ground for their own nests or stealing them from other nests (Carrascal et al. 1995; Moreno et al. 1995). The intensity of stone collection and theft of nest material have been demonstrated to be positively related to increases in the size of an individual's own nest (Moreno et al. 1995, 1999; Fargallo et al. 2001) and negatively associated with the packed redblood cell volume of parents, revealing the costly nature of this behaviour (Fargallo et al. 2001). Additionally, nest size is an important factor determining breeding success of pygoscelid penguins (Moreno et al. 1995). (2) A large body size allows a maximisation of the food carried by parent penguins to their chicks, allowing larger individuals (individuals with larger flipper length) to provide their chicks with larger meals per feeding visit (de Leon et al. 1998). (3) Breeding time is a life-history trait profoundly affecting breeding success in birds (Price et al. 1988). Chinstrap penguins are no exception, as earlier breeders produce larger and healthier chicks than late breeders (Vinuela et al. 1996; Moreno et al. 1997).

\section{Materials and methods}

\section{Study area and penguin sampling}

The study was made in the colony of Vapour Col, Deception Is-

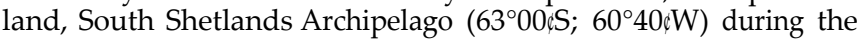
austral 1998-1999 breeding season. As the study started with the clutches already being incubated, breeding phenology was instead estimated using hatching dates. The hatching date was defined as the first date in which any of the chicks was observed fully or partially out of the egg. Brood sex ratios and nest size were estimated in a large subcolony (aggregates of nests separated from other such aggregates by areas unused for nesting) of 1,000 nests. Only nests with the modal clutch size of two eggs (Vinuela et al. 1996) were considered. Both chicks were sampled for sexing (see below) before mortality occurred (2-6 days after hatching). The fate of the nests sampled was not followed after blood sampling. Piles of stones forming the nests adopt irregular shapes making it difficult to determine the size of the nest by means of length measurements. However, the total amount of stones in a nest can be easily collected and separated from the cup scraped by penguins on the ground. Nest weight is a reliable measurement of nest size (Carrascal et al. 1995; Moreno et al. 1995) and parental workload (Moreno et al. 1995; Fargallo et al. 2001). The facility to obtain stones can vary depending on the position of the nest inside the subcolony (Carrascal et al. 1995); for this reason, all nests $(n=37)$ were randomly selected along two axes, the first extending from the border closest to the sea to the opposite side, and a second axis perpendicular to the first. This procedure was carried out in order to procure a similar availability of stones for all pairs. To measure nest weight, we removed the incubating parents and eggs (temporarily put in a warm bag during manipulation) and weighed (to the nearest $0.1 \mathrm{~kg}$ ) all stones in their nests. All stones were then returned to the nests and carefully arranged. No penguin abandonment was noted during or immediately after nest manipulations (see Fargallo et al. 2001 for further details).

In addition to the most intensively studied large subcolony (L), we also sampled 2 nearby subcolonies of 34 (S1) and 40 (S2) nests. In both small subcolonies, we sampled all broods in which both chicks hatched (18 and 20, respectively). Since parents from subcolonies L and S1 were sampled during the same breeding season for other studies (see Fargallo et al. 2001; Moreno et al. 2001), we used these data (flipper length of both parents) to search for associations between parental body size and brood sex ratio.

\section{Sex identification and validation}

A small drop of blood (20-50 11) was collected from all adults and hatchlings by means of foot-vein puncture and stored in ethanol for later sexing in the laboratory. DNA was prepared from whole blood using the Chelex-100 method (Walsh et al. 1991). The sexing method employed is that described by Griffiths and Tiwari (1995). PCR amplification was performed using a particular set of primers (2945F, cfR and 3224R; Ellegren 1996). The validity of results employing primers $2945 \mathrm{~F}, \mathrm{cfR}$ and $3224 \mathrm{R}$ was checked by sexing the same animals by SSCP analysis of amplified products using primers P2 and P3 (Griffiths and Tiwari 1995). All penguins (98 adults and 150 nestlings) showed the same result by using both methods.

Since the chinstrap penguin is a sexually size-dimorphic species in which males are larger than females (Amat et al. 1993), we considered for 49 pairs that the largest partner was male and smallest partner was female. The intra-pair difference in size was determined using the scores extracted from a discriminant function including bill depth, bill length and flipper length (Amat et al. 1993). In 47 pairs, the largest partner showed only 1 fragment after agarose electrophoresis (CHD1Z), and the smallest partner showed 2 fragments (CHD1Z and CHD1W), as expected for males and females, respectively. In one of the sampled pairs, the male was smaller than the female, and in another pair both partners were females. Therefore, by using the sexual dimorphic phenotype we could correctly sex $96 \%$ of breeding individuals.

\section{Statistical analyses}

We performed multivariate analyses to explore the effects of environmental and parental characteristics on offspring sex ratio. We fitted all the explanatory variables to the observed data using generalised linear mixed models (GLIMMIX, Littell et al. 1996) in SAS statistical software (SAS Institute, Cary, N.C.). We considered that chicks from the same nest were not independent samples. Therefore, nests were treated as random effect using a randomised complete block design, in which variation in each chick variable was controlled for nest and the remaining explanatory variables. Since sex of chicks showed a clear binomial distribution (Kolmogorov-Smirnov, $\mathrm{d}=0.004, \mathrm{P}=0.88$ ), we used the binomial error and logit link function. As explanatory variables, we considered: hatching date and parental male and female body size (flipper length). Variation in breeding performance (nest size, laying date or breeding success) has been observed to be associated with the size of the subcolony (Barbosa et al. 1997), and we therefore included the subcolony in the model as a random factor. Some of the explanatory variables could covary, and thus we fitted their effects to the observed data following forward stepwise procedure, testing the significance of each variable one by one, and adding only the variable that resulted in the largest increase in model fit. The result is the most adequate model for explaining the variability in the response variable, where only significant explanatory variables are retained. To assess the possible association between nest size and hatching sex ratio, we used only the colony in which the nests were measured (L). The cubic-root transformation was used for nest weight, although for descriptive purposes, untransformed values are presented in the figures.

\section{Results}

The hatchling sex ratio from 75 nests (150 nestlings) sampled was 0.54 , which did not differ significantly from 
parity (binomial test, $\mathrm{P}=\mathrm{O} .37$ ), although the statistical power of the test $(14 \%)$ was low. Large sample sizes are required to assess offspring sex biases at hatching. Considering all subcolonies, the GLIMMIX showed that hatchling sex ratio did not differ significantly among subcolonies and was not affected by hatching date or hatching order, with no significant interactions among factors (all $\mathrm{P}>0.14$ ). Considering the two subcolonies from which we have parental body measurements, the GLIMMIX showed that offspring sex ratio was not significantly explained by any of the variables we measured (all $\mathrm{P}>0.23$ ). No interactions among variables were found (all $\mathrm{P}>0.2$ ).

When we took into account the subcolony in which the size of nests was measured (L), the GLIMMIX showed that variation in the offspring sex ratio was only significantly explained by nest size $\left(\mathrm{F}_{1,36}=6.77\right.$, $\mathrm{P}=0.013$, Fig. 1). The power of the test $(73 \%)$ was high enough to support the hypothesis.

\section{Discussion}

The sex ratio at hatching was unrelated to breeding date or body size of chinstrap-penguin parents. Instead, brood sex ratio at hatching became more male-biased with increasing nest size. Earlier studies have shown a clear connection between nest size and reproductive success in chinstrap penguins, so that large nests improve nest insulation and small nests are more likely to suffer from flooding and subsequent high chick mortality (Moreno et al. 1995). Nest size is positively related to the intensity of stone-provisioning behaviour during the incubation and brooding periods (Carrascal et al. 1995; Moreno et al. 1995). The stone-provisioning activity dramatically increases when the risk of perceived nest flooding is experimentally increased (Fargallo et al. 2001). Furthermore, individuals working harder to provision their nests with stones during the incubation/ brooding period show lowered haematocrits, supporting

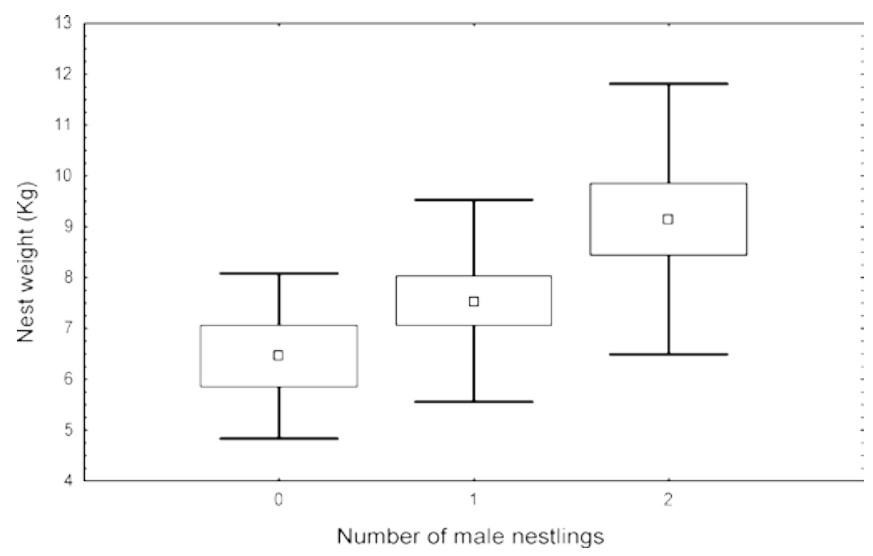

Fig. 1 Association between the number of male offspring and nest size (measured as nest weight). Boxes and whiskers represent $1 \mathrm{SE}$ and $1 \mathrm{SD}$, respectively the hypothesis that a trade-off in time and/or energy exists between stone-provisioning and health status, so that only individuals in prime condition are able to build and maintain large nests (Fargallo et al. 2001). Several pieces of experimental and observational evidence suggest therefore that nest size is an honest signal of parental quality in chinstrap penguins. Nest-building activities may be considered sexual displays, allowing each sex to reliably obtain information on the condition of the other, and willingness to invest in reproduction (Palomino et al. 1998; Soler et al. 1998, 2001). The "good parent process" of sexual selection is compatible with the functional explanation of nest size in pygoscelids (preventing flooding) as both operate at complementary levels of evolutionary explanation (Fargallo et al. 2001). This being the case, the question then becomes, why do parents of better quality produce more sons?

Chinstrap penguins are considered a monogamous species, at both social and genetic levels, and promiscuity has not been observed in our study colony (Moreno et al. 2001). This suggests that, in our population, the reproductive value of sons versus daughters is not different in this respect and should not be a concern in the search for adaptive explanations of sex ratios. However, female-biased mortality in pre-breeding and adult penguins has been observed in yellow-eyed penguins (Megadyptes antipodes) (Richdale 1957 in Williams 1995), little penguins (Eudyptula minor) (Dann and Cullen 1990), and in the closely related Adelie penguin (P. adeliae) (Ainley et al. 1983). A male-biased sex ratio appears to be characteristic of breeding populations in many penguin species (Williams 1995). This may reflect the fact that males delay the onset of breeding for 1 year longer than females, and they breed less frequently. In pygoscelid penguins, males arrive at the breeding colonies earlier than females and remain at the colony until egg laying (Trivelpiece and Trivelpiece 1990). During this period and afterwards, male-male competition for nest sites and mates occurs (Spurr 1974; Davis and Speirs 1990). Intense competition among males is probably due to female-biased mortality. In Adelie penguins, a proportion of 1.5 males to every female in breeding individuals has been observed, so that on average, only 2 out of 3 males will get a mate every breeding season. These males are expected to be those of higher quality.

Life-history theory predicts that for long-life iteroparous species, such as the chinstrap penguin, the reproductive value of an individual is determined by the "lifetime reproductive success", a major parameter defining fitness (Stearns 1992). If the survival prospects of females are lower, it can be expected, in principle, their reproductive value to be lower than for males. If a high-quality male survives better (because of being male) and is capable of frequently getting a partner (because of being of high-quality), this quality-sex class should be the best offspring option for parents. However, male chinstrap penguins are $5.6 \%$ heavier $(4.3 \%$ at 
pre-mating, $1.1 \%$ at copulation, $4.2 \%$ after clutch completion and $12.7 \%$ during chick-rearing periods) and 3\% larger (flipper length) than females (Williams 1995). Assuming that this degree of sexual-size dimorphism is large enough to induce a significant difference in food resource demands, only parents of better quality could rear high-quality sons. Our results concerning offspring sex ratio and parental quality may be similar to the views given by Trivers and Willard (1973), since parents apparently were able to modify the offspring sex ratio according to their own quality. Parents in better condition might invest more in sons than in daughters as the potential for fitness returns of producing sons would be higher by increasing the probability of survival and breeding of their progeny.

An increasing number of recent studies have demonstrated that females of some birds have biased offspring sex ratios at laying (Blanco et al. 2002; Hasselquist and Kempenaers 2002; Komdeur and Pen 2002), so that the control of the sex ratio of their offspring has been performed inside the mother. The physiological mechanisms by which bird mothers can adjust the sex of their offspring at fertilisation (primary sex ratio) according to the external and social environment or parental quality remain largely unknown (Komdeur and Pen 2002). It has been argued that maternal hormones might influence the sex ratio in birds (Krackow 1995; Williams 1999) and that female birds are able to vary the amount of hormones they can produce in relation to male attractiveness (Gil et al. 1999).

In summary, our results illustrate the connection between an indicator of parental quality and hatchling sex ratio and, in the context of recent life-history and sex allocation theories, agree with the suggestion that organisms such as birds can adjust the sex ratio of their offspring in order to increase their fitness in relation to sex-biased mortality and mating success. This study suggests a promising research avenue to understand sexratio variation by experimentally manipulating nest size prior to egg laying.

Acknowledgements We thank J. Moreno for comments, and the military and scientific staff of the Antarctic Spanish Base "Gabriel de Castilla" for their invaluable support during our stay at Deception Island. Transport to and from the island by the ship of the Spanish Navy "Hesperides" is gratefully acknowledged. This is a contribution to project ANT98-1443-E CICYT. The Spanish Antarctic Research Program C.I.C.Y.T gave permission to work in the study area and for penguin manipulations.

\section{References}

Ainley DG, LeResche RE, Sladen WJL (1983) Breeding biology of the Adelie penguin. University of California Press, Berkeley

Amat JA, Vinuela J, Ferrer M (1993) Sexing chinstrap penguins (Pygoscelis antarctica) by morphological measurements. Colon Waterbirds 16:213-215

Barbosa A, Moreno J, Potti J, Merino S (1997) Breeding group size, nest position and breeding success in the chinstrap penguin. Polar Biol 18:410-414
Blanco G, Davila JA, Lopez-Septiem JA, Rodriguez R, Martınez F (2002) Sex-biased initial eggs favours sons in the slightly sizedimorphic scops owl (Otus scops). Biol J Linn Soc 76:1-8

Bradbury RB, Cotton PA, Wright J, Griffiths R (1997) Nestling sex ratio in the European starling Sturnus vulgaris. J Avian Biol 28:255-258

Carrascal LM, Moreno J, Amat JA (1995) Nest maintenance and stone theft in the chinstrap penguin (Pygoscelis antarctica). 2. Effects of breeding group size. Polar Biol 15:541-545

Charnov EL (1982) The theory of sex allocation. Princeton University Press, N J

Dann P, Cullen JM (1990) Survival, patterns of reproduction, and lifetime reproductive output in little blue penguins (Eudyptula minor) on Phillip Island, Victoria, Australia. In: Davis LS, Darby JT (eds) Penguin biology. Academic, New York, pp 6384

Davis LS, Speirs EAH (1990) Mate choice in penguins. In: Davis LS, Darby JT (eds) Penguin biology. Academic, New York, pp 345-376

Ellegren H (1996) First gene on the avian W chromosome (CHD) provides a tag for universal sexing of non-ratite birds. Proc $R$ Soc Lond B 263:1635-1641

Fargallo JA, Leon A de, Potti J (2001) Nest maintenance effort and health status in chinstrap penguins, Pygoscelis antarctica: the functional significance of stone provisioning behaviour. Behav Ecol Sociobiol 50:141-150

Gil D, Graves J, Hazon N, Wells A (1999) Male attractiveness and differential testosterone investment in zebra finch eggs. Science 286:126-128

Griffiths R, Tiwari B (1995) Sex of the last wild spix's macaw. Nature 375:454

Hamilton WD (1967) Extraordinary sex ratios. Science 156:477_ 488

Hasselquist D, Kempenaers B (2002) Parental care and adaptive brood sex ratio manipulation in birds. Philos Trans $\mathrm{R}$ Soc Lond B 357:363-372

Koenig WD, Dickinson JL (1996) Nestling sex ratio variation in western bluebirds. Auk 113:902-910

Komdeur J, Pen I (2002) Adaptive sex allocation in birds: the complexities of linking theory and practice. Philos Trans R Soc Lond B 357:373-380

Krackow S (1995) Potential mechanisms for sex ratio adjustment in mammals and birds. Biol Rev 70:225-241

Leon A de, Fargallo JA, Moreno J (1998) Parental body size affects meal size in the chinstrap penguin (Pygoscelis antarctica). Polar Biol 19:358-360

Littell RC, Milliken GA, Stroup WW, Wolfinger RD (1996) SAS (R) system for mixed models. SAS Institute, Cary, N.C.

Moreno E, Moreno J, Leon A de (1999) The effect of nest size on stone gathering behaviour in the chinstrap penguin. Polar Biol 22:90-92

Moreno J, Bustamante J, Vinuela J (1995) Nest maintenance and stone theft in the chinstrap penguin (Pygoscelis antarctica). 1. Sex roles and effects on fitness. Polar Biol 15:533-540

Moreno J, Barbosa A, Potti J, Merino S (1997) The effects of hatching date and parental quality on chick growth and creching age in the chinstrap penguin Pygoscelis antarctica: a field experiment. Auk 114:47-54

Moreno J, Boto L, Fargallo JA, Leon A de, Potti J (2001) Absence of extra-pair fertilisations in the chinstrap penguin Pygoscelis antarctica. J Avian Biol 31:580-583

Muller-Schwarze D (1984) The behaviour of penguins. State University of New York Press, Albany

Newton I, Marquiss M (1979) Sex ratio among nestlings of the European sparrowhawk. Am Nat 113:309-315

Palomino JJ, Martın-Vivaldi M, Soler M, Soler JJ (1998) Functional significance of nest size variation in the rufous bush robin Cercotrichas galactotes. Ardea 86:177-185

Plagiani AC, Lee PL, Bradbury RB (1999) Molecular determination of sex ratio in yellowhammer Emberiza citrinella offspring. J Avian Biol 30:239-244 
Price T, Kirkpatrick M, Arnold S (1988) Directional selection and the evolution of breeding date in birds. Science 240:798799

Sheldon BC (1998) Recent studies of avian sex ratios. Heredity 80:397-402

Soler JJ, Møller AP, Soler M (1998) Nest building, sexual selection and parental investment. Evol Ecol 12:427-441

Soler JJ, Neve L de, Martınez JG, Soler M (2001) Nest size affects clutch size and the start of incubation in magpies: an experimental study. Behav Ecol 12:301-307

Spurr EB (1974) Individual differences in the aggressiveness of Adelie penguins. Anim Behav 22:611-616

Stearns SC (1992) The evolution of life histories. Oxford University Press, Oxford

Trivelpiece WZ, Trivelpiece SG (1990) Courtship period of Adelie, gentoo and chinstrap penguins. In: Davis LS, Darby JT (eds) Penguin biology. Academic, New York, pp 113-128
Trivers RL, Willard DE (1973) Natural selection of parental ability to vary the sex ratio of offspring. Science 179:90-92

Vinuela J, Moreno J, Carrascal LM, Sanz JJ, Amat JA, Ferrer M, Belliure J, Cuervo JJ (1996) The effect of hatching date on parental care, chick growth, and chick mortality in the chinstrap penguin Pygoscelis antarctica. J Zool Lond 240:51-58

Walsh D, Metzger A, Higuchi R (1991) Chelex 100 as a medium for simple extraction of DNA for PCR-based typing from forensic material. BioTechniques 10:506-513

Westerdahl H, Bensch S, Hansson B, Hasselquist D, von Schantz T (1997) Sex ratio variation among broods of great reed warblers Acrocephalus arundinaceus. Mol Ecol 6:543-548

Williams TD (1995) The penguins. Oxford University Press, Oxford

Williams TD (1999) Parental and first generation effects of exogenous 17beta-estradiol on reproductive performance of female zebra finches (Taeniopygia guttata). Horm Behav 35:135-143 